M. Itô and N. Suzuki

Nagoya Math. J.

Vol. 99 (1985), 89-110

\title{
THE SEMI-BALAYABILITY OF REAL CONVOLUTION KERNELS
}

\author{
MASAYUKI ITÔ AND NORIAKI SUZUKI \\ Dedicated to Professor Yukio Kusunoki on his 60th birthday
}

\section{$\S 1$.}

Let $X$ be a locally compact, $\sigma$-compact and non-compact abelian group. Throughout this paper, we shall denote by $\xi$ a fixed Haar measure on $X$ and by $\delta$ the Alexandroff point of $X$.

A real convolution kernel (i.e., a real Radon measure) $N$ on $X$ is said to be semi-balayable if $N$ satisfies the semi-balayage principle on all open sets (see Definition 6). We know that every convolution kernel $N$ of logarithmic type is semi-balayable (see [8]). Here $N$ is said to be of logarithmic type if, with a vaguely continuous, markovian, semi-transient and recurrent convolution semi-group $\left(\alpha_{t}\right)_{t \geqq 0}$ of non-negative Radon measures on $X$,

$$
N * \mu=\int_{0}^{\infty} \alpha_{t} * \mu d t\left(=\lim _{t \rightarrow \infty} \int_{0}^{t} \alpha_{s} * \mu d s^{1)}\right)
$$

for all real Radon measure $\mu$ on $X$ with compact support and $\int d \mu=0$.

In this paper, we shall show that the semi-balayability is an essential property to characterize convolution kernels of logarithmic type. More precisely, we shall establish the following theorems.

TheOREM 1. Let $N$ be a real convolution kernel on $X$. If $X \approx R \times F$ or $X \approx Z \times F$, we suppose an additional condition: $N=o(|x|)$ at the infinity $^{2)}$. Then $N$ is of logarithmic type if and only if $N$ is semi-balayable, non-periodic and satisfies $\inf _{x \in X} N * f(x) \leqq 0$ for any finite continuous function $f$ on $X$ with compact support and $\int f d \xi=0$.

Received May 28, 1984.

1) For a net $\left(\mu_{\alpha}\right)_{\alpha \in \Lambda}$ of real Radon measures and a real Radon measure $\mu$, we write $\mu=\lim _{\alpha \in \Lambda} \mu_{\alpha}$ if $\left(\mu_{\alpha}\right)_{\alpha \in \Lambda}$ converges vaguely to $\mu$ along $\Lambda$.

2) If $X=R \times F$ or $X=Z \times F, N=o(|x|)$ at the infinity means that for any $f \in$ $C_{K}^{+}(X), N * f((x, y))=o(|x|)$ as $|x| \rightarrow \infty$, where $(x, y) \in R \times F$ or $\in Z \times F$. In the case of $X \approx R \times F$ or $X \approx Z \times F$, the definition $N=o(|x|)$ at the infinity follows naturally from the above definition. 
Here $R, Z$ and $F$ denote the additive group of real numbers, the additive group of integers and a certain compact abelian group, respectively.

By virtue of the main theorems in [8] (see Théorèmes 52 and 52'), Theorem 1 follows immediately from the following

TheOREM 2. Let $N$ be a non-periodic real convolution kernel on $X$ satisfying $\inf _{x \in X} N * f(x) \leqq 0$ for any finite continuous function $f$ on $X$ with compact support and $\int f d \xi=0$. Then $N$ is semi-balayable if and only if $N$ satisfies the semi-complete maximum principle and $\eta_{N, \delta}=-\infty$, i.e., for any exhaustion $\left(K_{n}\right)_{n=1}^{\infty}$ of $X^{3)}$ and any non-negative continuous function $f \neq 0$ on $X$ with compact support, $\lim _{n \rightarrow \infty} \int f d \eta_{N, C K_{n}}=-\infty$, where $\eta_{N, C K_{n}}$ is the $N$-reduced measure of $N$ on $C K_{n}$.

The "if" part is already known (see Proposition 28 in [8]), so that this paper will be devoted principally to the proof of the "only if" part.

It is interesting to compare Theorem 1 with the Choquet-Deny theorem for Hunt convolution kernels ${ }^{4}$ (see [3]).

Contrary to a conjecture in [8] (see Problème 29), Theorem 1 shows that, under some additional conditions, non-periodic and semi-balayable real convolution kernels are of logarithmic type.

$\S 2$.

We denote by:

$C(X)$ the usual Fréchet space of finite continuous functions on $X$;

$C_{K}(X)$ the usual topological vector space of finite continuous functions on $X$ with compact support;

$M(X)=C_{K}(X)^{*}$ the topological vector space of real Radon measures on $X$ with the vague (weak*) topology;

$M_{K}(X)=C(X)^{*}$ the usual topological vector space of real Radon measures on $X$ with compact support;

$C^{+}(X), \quad C_{K}^{+}(X), \quad M^{+}(X)$ and $M_{K}^{+}(X)$ their subsets of non-negative elements.

Furthermore, we put

$$
C_{K}^{0}(X)=\left\{f \in C_{K}(X) ; \int f d \xi=0\right\} \quad \text { and } \quad M_{K}^{0}(X)=\left\{\mu \in M_{K}(X) ; \int d \mu=0\right\} .
$$

3) An exhaustion $\left(K_{n}\right)_{n=1}^{\infty}$ of $X$ means a sequence of compact sets satisfying $K_{n} \subset$ the interior of $K_{n+1}$ and $\cup_{n=1}^{\infty} K_{n}=X$.

4) A non-negative convolution kernel $N_{0}$ on $X$ is a Hunt convolution kernel if and only if $N_{0}$ is balayable (see Remark 14 (3)) and not pseudo-periodic. 
Definition 3. A real convolution kernel $N$ on $X$ is said to satisfy the semi-complete maximum principle (denoted by $N \in$ (SMP)) if for any $f, g \in C_{K}^{+}(X)$ with $\int f d \xi=\int g d \xi$ and any $a \in R$, we have the implication:

$$
N * f(x) \leqq N * g(x)+a \quad \text { on } \operatorname{supp}(f) \Longrightarrow N * f(x) \leqq N * g(x)+a \quad \text { on } X,
$$

where $\operatorname{supp}(f)$ denotes the support of $f$.

Definition 4. A real convolution $N$ on $X$ is said to satisfy the transitive semi-complete maximum principle with respect to $\xi$ (denoted by $(N, \xi) \in(\mathrm{TSMP}))$ if for any $f, g \in C_{K}^{+}(X)$ with $\int f d \xi=\int g d \xi$ and any $a \in R$, we have the implication:

$$
N * f(x) \leqq N * g(x)+a \quad \text { on } \operatorname{supp}(f) \Longrightarrow a \geqq 0 .
$$

We can describe the above principles by the term of non-negative Radon measures.

Remark 5. $N \in(\mathrm{SMP})$ (resp. $(N, \xi) \in(\mathrm{TSMP})$ ) if and only if for any $\mu, \nu \in M_{K}^{+}(X)$ with $\int d \mu=\int d \nu$ and any $a \in R$, we have the implication:

$$
\begin{gathered}
N * \mu \leqq N * \nu+a \xi \quad \text { in a certain open set } \supset \operatorname{supp}(\mu) \\
\Longrightarrow N * \mu \leqq N * \nu+a \xi \quad \text { on } X(\text { resp. } \Longrightarrow a \geqq 0),
\end{gathered}
$$

where $\operatorname{supp}(\mu)$ denotes also the support of $\mu$.

For a real convolution kernel $N$ on $X$, we put

$$
D^{+}(N)=\left\{\mu \in M^{+}(X) ; N * \mu \text { is defined in } M(X)\right\} \text {. }
$$

Let $\mu \in M^{+}(X)$. Evidently $\mu \in D^{+}(N)$ if and only if for any $f \in C_{K}^{+}(X)$, $\int|\check{N} * f| d \mu<\infty$. Here $\check{N}$ denotes the real convolution kernel on $X$ defined by $\int f d \check{N}=\int \check{f} d N$ for all $f \in C_{K}(X)$, where $\check{f}(x)=f(-x)$.

Definition 6. A real convolution kernel $N$ on $X$ is said to satisfy the semi-balayage principle (resp. the semi-balayage principle on all open sets) (denoted by $N \in(\mathrm{SBP})$ (resp. denoted by $N \in\left(\mathrm{SBP}_{g}\right)$ )) if for any $\mu \in M_{K}^{+}(X)$, any $a \in R$ and any relatively compact open set (resp. any open set) $\omega \neq \phi$ in $X$, there exists an element $\left(\mu^{\prime}, a^{\prime}\right) \in M^{+}(X) \times R$ such that:
(B.1) $\int d \mu^{\prime}=\int d \mu$.
(B.2) $\operatorname{supp}\left(\mu^{\prime}\right) \subset \bar{\omega}$. 
(B.3) $\mu^{\prime} \in D^{+}(N)$ and $N * \mu^{\prime}+a^{\prime} \xi=N * \mu+a \xi$ in $\omega$.

(B.4) $N * \mu^{\prime}+a^{\prime} \xi \leqq N * \mu+a \xi$ on $X$.

In this case, we call $\left(\mu^{\prime}, a^{\prime}\right)$ a semi-balayaged couple of $(\mu, a)$ on $\omega$ with respect to $N$ and denote by $\operatorname{SB}_{N}((\mu, a) ; \omega)$ the totality of such couples. If $N \in\left(\mathrm{SBP}_{g}\right)$, we say that $N$ is semi-balayable.

We set

$$
\begin{aligned}
\underline{\operatorname{SB}}_{N}((\mu, a) ; \omega) & =\left\{\left(\mu^{\prime}, a^{\prime}\right) \in \mathrm{SB}_{N}((\mu, a) ; \omega) ; N * \mu+a^{\prime} \xi\right. \\
& \left.=\inf \left\{N * \mu^{\prime \prime}+a^{\prime \prime} \xi ;\left(\mu^{\prime \prime}, a^{\prime \prime}\right) \in \operatorname{SB}_{N}((\mu, a) ; \omega)\right\}^{5)}\right\} .
\end{aligned}
$$

When $\bar{\omega}$ is non-compact, it is not easy to examine directly whether $\underline{\mathrm{SB}}_{N}((\mu, a) ; \omega) \neq \phi$ or $=\phi$.

Let $N \in(\mathrm{SBP})$ (resp. $N \in\left(\mathrm{SBP}_{g}\right)$ ). For $\mu \in D^{+}(N)$ with $\int d \mu<\infty, a \in R$ and a relatively compact open set (resp. an open set) $\omega \neq \phi$ in $X$, we can define $\mathrm{SB}_{N}((\mu, a) ; \omega)$ and $\underline{\mathrm{SB}}_{N}((\mu, a) ; \omega)$ analogously.

We shall use known results concerning potential theoretic principles for a real convolution kernel $N$ on $X$ (see Remarques 2, 7, Proposition 11 and Corollaire 14 in [8]).

Remark 7. (1) $N \in(\mathrm{SMP})$ and $N \in(\mathrm{SBP})$ are equivalent.

(2) Assume that $N \in(\mathrm{SMP})$. Then $(N, \xi) \in(\mathrm{TSMP})$ is equivalent to $\inf _{x \in X} N * f(x) \leqq 0$ for any $f \in C_{K}^{0}(X)$.

(3) Assume that $(N, \xi) \in($ TSMP). Then $N$ and $\check{N}$ satisfy the maximum principle, that is, for any $f \in C_{K}^{+}(X)$, we have $N * f(x) \leqq \sup _{y \in \operatorname{supp}(f)} N * f(y)$, on $X$ and $\check{N} * f(x) \leqq \sup _{y \in \operatorname{supp}(f)} \check{N} * f(y)$ on $X$.

Lemma 8. Let $N \in(\mathrm{SMP})$ and $\omega \neq \phi$ be a relatively compact open set in $X$. Then we have:

(1) For any $\mu \in D^{+}(N)$ with $\int d \mu<\infty$ and any $a \in R$, we have $\mathrm{SB}_{N}((\mu, a) ; \omega) \neq \phi$, and for any $\left(\mu^{\prime}, a^{\prime}\right) \in \underline{\mathrm{SB}}_{N}((\mu, a) ; \omega)$, there exist nets $\left(\mu_{\alpha}\right)_{\alpha \in A}$ in $M_{K}^{+}(X)$ and $\left(a_{\alpha}\right)_{\alpha \in \Lambda}$ in $R$ such that $\operatorname{supp}\left(\mu_{\alpha}\right) \subset \omega$ and $\left(N * \mu_{\alpha}+a_{\alpha} \xi\right)_{\alpha \in \Lambda}$ converges increasingly to $N * \mu^{\prime}+a^{\prime} \xi$ on $X$ along $\Lambda$.

(2) For $0<c \in R$, we denote by $\operatorname{SP}_{c}(N)$ the vague closure of

$$
\left\{N * \nu+a \xi ; \nu \in M_{K}^{+}(X), \int d \nu=c, a \in R\right\} .
$$

For any $\eta \in \operatorname{SP}_{c}(N)$, there exists an element $\left(\mu^{\prime}, a^{\prime}\right) \in M_{K}^{+}(X) \times R$ such that

5) This means that $\inf \left\{N * \mu^{\prime \prime}+a^{\prime \prime} \xi ;\left(\mu^{\prime \prime}, a^{\prime \prime}\right) \in \mathrm{SB}_{N}((\mu, \alpha) ; \omega)\right\}$ exists as a real Radon measure on $X$ and it is equal to $N * \mu^{\prime}+a^{\prime} \xi$. 
$\int d \mu^{\prime}=c, \operatorname{supp}\left(\mu^{\prime}\right) \subset \bar{\omega}, N * \mu^{\prime}+a^{\prime} \xi=\eta$ in $\omega$ and $N * \mu^{\prime}+a^{\prime} \xi \leqq \eta$ on $X$.

Proof. The assertion (1) is proved in the same manner as in [8] (see Corollaire 12). We shall show the assertion (2). We choose nets $\left(\mu_{\alpha}\right)_{\alpha \in \Lambda}$ in $M_{K}^{+}(X)$ with $\int d \mu_{\alpha}=c$ and $\left(a_{\alpha}\right)_{\alpha \in \Lambda}$ in $R$ such that $\lim _{\alpha \in \Lambda}\left(N * \mu_{\alpha}+a_{\alpha} \xi\right)$ $=\eta$. Let $\left(\mu_{\alpha}^{\prime}, a_{\alpha}^{\prime}\right) \in \operatorname{SB}_{N}\left(\left(\mu_{\alpha}, a_{\alpha}\right) ; \omega\right)$. Since $\int d \mu_{\alpha}^{\prime}=\int d \mu_{\alpha}=c$, we may assume that $\left(\mu_{\alpha}^{\prime}\right)_{\alpha \in \Lambda}$ converges vaguely. Put $\mu^{\prime}=\lim _{\alpha \in \Lambda} \mu_{\alpha}^{\prime}$. All $\mu_{\alpha}^{\prime}$ being supported by the compact set $\bar{\omega}$, we have $N * \mu^{\prime}=\lim _{\alpha \in A} N * \mu_{\alpha}^{\prime}$. This implies. that $\left(a_{\alpha}^{\prime}\right)_{\alpha \in \Lambda}$ converges. Putting $a^{\prime}=\lim _{\alpha \in \Lambda} a^{\prime}$, we see that $\left(\mu^{\prime}, a^{\prime}\right)$ is a required element.

We shall use a more general form of the semi-complete maximum principle.

Proposition 9. Let $N \in(\mathrm{SMP}),(N, \xi) \in(\mathrm{TSMP}), \mu \in D^{+}(N)$ with $c=$ $\int d \mu<\infty, a \in R$ and let $\eta \in \operatorname{SP}_{c}(N)$. If $N * \mu+a \xi \leqq \eta$ in a certain open set containing supp $(\mu)$, then the same inequality holds on $X$.

For the proof of this proposition, we shall use the following known lemma.

Lemma 10 (see Lemme 21 in [8]). Let $N \in(\mathrm{SMP})$ and $\left(\mu_{\alpha}\right)_{\alpha \in A}$ be a net in $M_{K}^{+}(X)$. If $\lim _{\alpha \in \Lambda} \int d \mu_{\alpha}=0$ and $\left(N * \mu_{\alpha}\right)_{\alpha \in \Lambda}$ converges vaguely, then there exists $b \in R$ such that $\lim _{\alpha \in \Lambda} N * \mu_{\alpha}=b \xi$. Furthermore, if $(N, \xi) \in$ (TSMP), then $b \leqq 0$.

Proof of Proposition 9. If $\mu \in M_{K}^{+}(X)$, then our assertion follows from Remark 5 and Lemma 8. In general case, we choose an open exhaustion $\left(\omega_{n}\right)_{n=1}^{\infty}$ of $X^{6}$. Let $\omega$ be an open set in $X$ satisfying $\omega \supset \operatorname{supp}(\mu)$ and $N * \mu+a \xi \leqq \eta$ in $\omega$. We may assume that $\omega \cap \omega_{1} \neq \phi$. Put $\mu_{n}=\mu_{\omega_{n}}{ }^{7}$ and $\lambda_{n}=\mu-\mu_{n}$. Let $\left(\lambda_{n}^{\prime}, a_{n}^{\prime}\right) \in \underline{\mathrm{SB}}_{N}\left(\left(\lambda_{n}, a\right) ; \omega \cap \omega_{n}\right)$. Then $\left(\mu_{n}+\lambda_{n}^{\prime}, a_{n}^{\prime}\right) \in$ $\mathrm{SB}_{N}\left((\mu, a) ; \omega \cap \omega_{n}\right)$, and Lemma 8 (1) gives

$$
N *\left(\mu_{n}+\lambda_{n}^{\prime}\right)+a_{n}^{\prime} \xi \leqq \eta \quad \text { on } X .
$$

Hence it suffices to show that $\lim _{n \rightarrow \infty}\left(N *\left(\mu_{n}+\lambda_{n}^{\prime}\right)+a_{n}^{\prime} \xi\right)=N * \mu+a \xi$.

6) An open exhaustion $\left(\omega_{n}\right)_{n=1}^{\infty}$ of $X$ means a sequence of relatively compact open sets $\neq \phi$ in $X$ satisfying $\omega_{n+1} \supset \bar{\omega}_{n}$ and $\cup_{n=1}^{\infty} \omega_{n}=X$.

7) Far $\mu \in M(X)$ and a universally measurable set $E$ in $X,\left.\mu\right|_{E}$ denotes the real Radon measure on $X$ defined by $\left.\mu\right|_{E}=\mu$ on $E$ and $\left.\mu\right|_{E}=0$ on $C E$. 
From $(N, \xi) \in$ (TSMP), we see that $a_{n}^{\prime} \leqq a_{n+1}^{\prime} \leqq a$ for all $n \geqq 1$, so that $\left(N * \lambda_{n}^{\prime}\right)_{n=1}^{\infty}$ converges vaguely. By Lemma 10 and $\lim _{n \rightarrow \infty} \int d \lambda_{n}^{\prime}=0$, there exists $0 \geqq b \in R$ such that $\lim _{n \rightarrow \infty} N * \lambda_{n}^{\prime}=b \xi$. Since

$$
\lim _{n \rightarrow \infty} N *\left(\mu_{n}+\lambda_{n}^{\prime}\right)+\left(\lim _{n \rightarrow \infty} a_{n}^{\prime}\right) \xi=N * \mu+a \xi \quad \text { in } \omega,
$$

$\lim _{n \rightarrow \infty} a_{n}^{\prime}=a$ and $b=0$. Thus $N *\left(\mu_{n}+\lambda_{n}^{\prime}\right)+a_{n}^{\prime} \xi$ converges increasingly to $N * \mu+a \xi$ as $n \uparrow \infty$, which completes the proof.

Similarly we obtain the following

Proposition 11. Let $N \in\left(\mathrm{SBP}_{g}\right)$ and $(N, \xi) \in(\mathrm{TSMP})$. Then, for any $\mu \in M_{K}^{+}(X)$, any $a \in R$, any open set $\omega \neq \phi$ in $X$ and any $\left(\mu^{\prime}, a^{\prime}\right) \in \mathrm{SB}_{N}((\mu, a) ; \omega)$, we have $a^{\prime} \leqq a$. Furthermore, if $C \omega$ is compact, $a^{\prime}=a$.

Proof. Let $\left(\omega_{n}\right)_{n=1}^{\infty}$ be an open exhaustion of X. Put $\mu_{n}^{\prime}=\left.\mu^{\prime}\right|_{\omega_{n}}$ and $\lambda_{n}-$ $\mu^{\prime}-\mu_{n}^{\prime}$. Choose $\left(\lambda_{n}^{\prime}, a_{n}^{\prime}\right) \in \underline{\mathrm{SB}}_{N}\left(\left(\lambda_{n}, a^{\prime}\right) ; \omega_{n}\right)$; then $\left(\mu_{n}^{\prime}+\lambda_{n}^{\prime}, a_{n}^{\prime}\right) \in \underline{\mathrm{SB}}_{N}\left(\left(\mu^{\prime}, a^{\prime}\right) ; \omega_{n}\right)$. Then $(N, \xi) \in\left(\right.$ TSMP) gives $a_{n}^{\prime} \leqq a$. From the above proof, we see that $\lim _{n \rightarrow \infty} a_{n}^{\prime}=a^{\prime}$, that is, $a^{\prime} \leqq a$.

The latter part is shown in the same manner as in Proposition 28 (2) in [8].

It is a question when $a^{\prime}=a$ holds.

$\S 3$.

In this paragraph, we shall prepare some potential theoretic results concerning shift-bounded Hunt convolution kernels.

Definition 12. A non-negative convolution kernel $N_{0}$ on $X$ is said to be a Hunt convolution kernel if it is of form

$$
\left.N_{0}=\int_{0}^{\infty} \alpha_{t} d t \text { (i.e., for any } f \in C_{K}(X), \int f d N_{0}=\int_{0}^{\infty} d t \int f d \alpha_{t}\right),
$$

where $\left(\alpha_{t}\right)_{t \geqq 0}$ is a vaguely continuous convolution semi-group (of positive Radon measures) on $X$, i.e., $\alpha_{0}=$ the unit measure $\varepsilon$ at the origin 0 , $\alpha_{t} * \alpha_{s}=\alpha_{t+s}$ for all $t \geqq 0, s \geqq 0$ and $t \rightarrow \alpha_{t}$ is vaguely continuous.

In this case, $\left(\alpha_{t}\right)_{t \geq 0}$ is uniquely determined (see [5]) and called the convolution semi-group of $N_{0}$.

A vaguely continuous convolution semi-group $\left(\alpha_{t}\right)_{t \geqq 0}$ is said to be sub-markovian (resp. markovian) if $\int d \alpha_{t} \leqq 1\left(\operatorname{resp} . \int d \alpha_{t}=1\right)$ for all $t \geqq 0$. 
Definition 13. A family $\left(N_{p}\right)_{p>0}$ of non-negative convolution kernels on $X$ is said to be a resolvent if for any $p>0$ and $q>0$,

$$
N_{p}-N_{q}=(q-p) N_{p} * N_{q} \text { (The resolvent equation). }
$$

A non-negative convolution kernel $N_{0}$ on $X$ possesses the resolvent if there exists a resolvent $\left(N_{p}\right)_{p>0}$ with $N_{0}=\lim _{p \downarrow 0} N_{p}$.

In this case, $N_{0}-N_{p}=p N_{0} * N_{p}$ and $\operatorname{supp}\left(N_{0}\right)=\operatorname{supp}\left(N_{p}\right) \quad(p>0)$ hold, and $\left(N_{p}\right)_{p>0}$ is uniquely determined (see [5]). We call it the resolvent of $N_{0}$.

A resolvent $\left(N_{p}\right)_{p>0}$ is said to be sub-markovian (resp. markovian) if for any $p>0, p \int d N_{p} \leqq 1$ (resp. $\left.p \int d N_{p}=1\right)$.

The following results are fundamental for Hunt convolution kernels (see [1], [3], [5], [6] and [7]).

Remark 14. (1) A non-negative convolution kernel $N_{0}$ on $X$ is a Hunt convolution kernel if and only if its resolvent exists and $N_{0}$ is non-periodic, i.e., for any $x \in X, N_{0} \neq N_{0} * \varepsilon_{x}$ provided with $x \neq 0$, where $\varepsilon_{x}$ denotes the unit measure at $x$.

(2) Let $N_{0}$ be a Hunt convolution kernel on $X$. Then the equivalences (a) $\Leftrightarrow(\mathrm{b}) \Leftrightarrow$ (c) hold:

(a) The convolution semi-group of $N_{0}$ is sub-markovian (resp. markovian).

(b) The resolvent of $N_{0}$ is sub-markovian (resp. markovian).

(c) $N_{0}$ is shift-bounded, i.e., for any $f \in C_{K}(X), N * f$ is bounded on $X$ (resp. shift-bounded and $\left.\int d N_{0}=\infty\right)$.

(3) Let $N_{0}$ be a shift-bounded Hunt convolution kernel on $X$. Then we have:

(a) (The balayability). For any $\mu \in M_{K}^{+}(X)$ and any open set $\omega$ in $X$, there exists $\mu^{\prime} \in D^{+}\left(N_{0}\right)$ such that $\operatorname{supp}\left(\mu^{\prime}\right) \subset \bar{\omega}, N_{0} * \mu^{\prime}=N_{0} * \mu$ in $\omega$ and $N_{0} * \mu^{\prime} \leqq N_{0} * \mu$ on $X$.

In this case, $\mu^{\prime}$ is called an $N_{0}$-balayaged measure of $\mu$ on $\omega$, and $\int d \mu^{\prime} \leqq d \mu$ holds. We have $\int d N_{0}=\infty$ if and only if, for any $\mu \in M_{K}^{+}(X)$, any open set $\omega$ in $X$ whose complement is compact and any $N_{0}$-balayaged measure $\mu^{\prime}$ of $\mu$ on $\omega, \int d \mu^{\prime}=\int d \mu$.

(b) (The complete maximum principle). For any $\mu, \nu \in M_{K}^{+}(X)$ and any $0 \leqq c \in R, N_{0} * \mu \leqq N_{0} * \nu+c \xi$ in a certain neighborhood of $\operatorname{supp}(\mu)$ 
implies that the same inequality holds on $X$.

(c) (The equilibrium principle). For any relatively compact open set $\omega$ in $X$, there exists $\gamma \in M_{K}^{+}(X)$ such that $\operatorname{supp}(\gamma) \subset \bar{\omega}, N_{0} * \gamma=\xi$ in $\omega$ and $N_{0} * \gamma \leqq \xi$ on $X$.

In this case, $\gamma$ is called an $N_{0}$-equilibrium measure of $\omega$.

(d) (The positive mass principle). For any $\mu, \nu \in M_{K}^{+}(X), N_{0} * \mu \leqq$ $N_{0} * \nu$ on $X$ implies $\int d \mu \leqq \int d \nu$.

(e) (The dominated convergence property). Let $\left(\mu_{\alpha}\right)_{\alpha \in \Lambda}$ be a net in $D^{+}\left(N_{0}\right)$ and $\mu \in M^{+}(X)$. If $\lim _{\alpha \in \Lambda} \mu_{\alpha}=\mu$ and there exists $\nu \in D^{+}\left(N_{0}\right)$ satisfying $N_{0} * \mu_{\alpha} \leqq N_{0} * \nu$ on $X$ for all $\alpha \in \Lambda$, then $\lim _{\alpha \in \Lambda} N_{0} * \mu_{\alpha}=N_{0} * \mu$.

(f) (The injectivity). For any $\mu, \nu \in D^{+}\left(N_{0}\right), N_{0} * \mu=N_{0} * \nu$ on $X$ implies $\mu=\nu$.

For $\mu \in D^{+}\left(N_{0}\right)$ and an open set $\omega$ in $X$, we can define analogously $N_{0}$-balayaged measures of $\mu$ on $\omega$ and denote by $\mathrm{B}_{N_{0}}(\mu ; \omega)$ their totality. It is well-known that $\mathrm{B}_{N_{0}}(\mu ; \omega) \neq \phi$. Put

$$
\mathrm{B}_{N_{0}}(\mu ; \omega)=\left\{\mu^{\prime} \in \mathrm{B}_{N_{0}}(\mu ; \omega) ; N_{0} * \mu^{\prime}=\inf \left\{N_{0} * \mu^{\prime \prime} ; \mu^{\prime \prime} \in \mathrm{B}_{N_{0}}(\mu ; \omega)\right\}\right\} \quad\left(\text { see }^{5)}\right)
$$

and

$$
\overline{\mathrm{B}}_{N_{0}}(\mu ; \omega)=\left\{\mu^{\prime} \in \mathrm{B}_{N_{0}}(\mu ; \omega) ; N_{0} * \mu^{\prime}=\sup \left\{N_{0} * \mu^{\prime \prime} ; \mu^{\prime \prime} \in \mathrm{B}_{N_{0}}(\mu ; \omega)\right\}\right\} \quad\left(\text { see }^{5)}\right) .
$$

For an open set $\omega$ in $X$, we can define analogously $N_{0}$-equilibrium measures of $\omega$ and denote by $\mathrm{E}_{N_{0}}(\omega)$ their totality. Put

$$
\underline{\mathrm{E}}_{N_{0}}(\omega)=\left\{\gamma \in \mathrm{E}_{N_{0}}(\omega) ; N_{0} * \gamma=\inf \left\{N_{0} * \gamma^{\prime} ; \gamma^{\prime} \in \mathrm{E}_{N_{0}}(\omega)\right\}\right\} \quad\left(\operatorname{see}^{5)}\right)
$$

provided with $\mathrm{E}_{N_{0}}(\omega) \neq \phi$.

Lemma 15. Let $N_{0}$ be a shift-bounded Hunt convolution kernel on $X$. Then we have:

(1) For any $\mu \in D^{+}\left(N_{0}\right)$ and any open set $\omega$ in $X, \underline{\mathrm{B}}_{N_{0}}(\mu ; \omega) \neq \phi$ and $\overline{\mathrm{B}}_{N_{0}}(\mu ; \omega) \neq \phi$. Moreover, $\underline{\mathrm{B}}_{N_{0}}(\mu ; \omega)$ and $\overline{\mathrm{B}}_{N_{0}}(\mu ; \omega)$ form only one element.

(2) For any $\mu \in D^{+}\left(N_{0}\right)$ and any two open sets $\omega_{1}, \omega_{2}$ in $X$ with $\omega_{1} \subset \omega_{2}$, we have $N_{0} * \mu_{1}^{\prime} \leqq N_{0} * \mu_{2}^{\prime}$ and $N_{0} * \mu_{1}^{\prime \prime} \leqq N_{0} * \mu_{2}^{\prime \prime}$ on $X$, where $\mu_{i}^{\prime} \in \underline{\mathrm{B}}_{N_{0}}\left(\mu ; \omega_{i}\right)$ and $\mu_{i}^{\prime \prime} \in \overline{\mathrm{B}}_{N_{0}}\left(\mu: \omega_{i}\right)(i=1,2)$.

(3) Put $P\left(N_{0}\right)=\left\{\mathrm{N}_{0} * \mu ; \mu \in D^{+}\left(N_{0}\right)\right\}$, where the closure is in the sense of the vague topology. For any $\mu \in D^{+}\left(N_{0}\right)$ and any $\eta \in P\left(N_{0}\right), N_{0} * \mu \leqq \eta$ in a certain open set $\supset \operatorname{supp}(\mu)$ implies that the same inequality holds on $X$. 
(4) For an open set $\omega$ in $X, \mathrm{E}_{N_{0}}(\mu) \neq \phi$ implies $\underline{\mathrm{E}}_{N_{0}}(\mu) \neq \phi$. In this case, $\underline{\mathrm{E}}_{N_{0}}(\mu)$ forms only one element.

(5) For $0<c \in R$, we put $P_{c}\left(N_{0}\right)=\overline{\left\{N_{0} * \mu ; \mu \in D^{+}\left(N_{0}\right), \int d \mu \leqq c\right\}}$. For any $\mu \in D^{+}\left(N_{0}\right)$ and any $\eta \in P_{c}\left(N_{0}\right), N_{0} * \mu \leqq \eta$ on $X$ implies $\int d \mu \leqq c$.

(6) Let $\left(\mu_{\alpha}\right)_{\alpha \in A}$ be a net in $D^{+}\left(N_{0}\right)$ and $0 \neq \lambda_{1}, 0 \neq \lambda_{2} \in M_{K}^{+}(X)$. If there exist $\nu \in D^{+}\left(N_{0}\right)$ and a relatively compact net $\left(x_{\alpha}\right)_{\alpha \in \Lambda}$ in $X$ such that $N_{0} * \mu_{\alpha} * \lambda_{1} \leqq N_{0} * \nu * \varepsilon_{x_{\alpha}} * \lambda_{2}$ on $X$, then $\left(\mu_{\alpha}\right)_{\alpha \in A}$ is vaguely bounded. If $\mu_{\alpha} \rightarrow$ $\mu \in M^{+}(X)$, then $\lim _{\alpha \in A} N_{0} * \mu_{\alpha}=N_{0} * \mu$.

Proof. (1) Let $\left(\omega_{\alpha}\right)_{\alpha \in A}$ be a net of open sets in $X$ with $\bar{\omega}_{\alpha} \subset \omega_{\beta}(\alpha \supseteqq \beta)$ and $\bigcup_{\alpha \in A} \omega_{\alpha}=\omega$. We choose $\mu_{\alpha}^{\prime} \in B_{N_{0}}\left(\mu ; \omega_{\alpha}\right)$. Then the complete maximum principle of $N_{0}$ implies that for any $\mu^{\prime \prime} \in \mathrm{B}_{N_{0}}(\mu ; \omega), N_{0} * \mu_{\alpha}^{\prime} \leqq N_{0} * \mu^{\prime \prime}$ on $X$. This and the dominated convergence property of $N_{0}$ show that $\left(\mu^{\prime}\right)_{\alpha \in \Lambda}$ is vaguely bounded and every vaguely accumulation point of $\left(\mu_{\alpha}^{\prime}\right)_{\alpha \in \Lambda}$ as $\omega_{\alpha} \uparrow \omega$ is contained in $\underline{\mathrm{B}}_{N_{0}}(\mu ; \omega)$, which gives $\underline{\mathrm{B}}_{N_{0}}(\mu ; \omega) \neq \phi$. Let $\left(\omega_{\alpha^{\prime}}^{\prime}\right)_{\alpha^{\prime} \in A^{\prime}}$ be a net of open sets in $X$ with $\omega_{\alpha^{\prime}}^{\prime} \supset \bar{\omega}_{\beta^{\prime}}^{\prime}\left(\alpha^{\prime} \supseteqq \beta^{\prime}\right)$ and $\bigcap_{\alpha^{\prime} \in A^{\prime}} \omega_{\alpha^{\prime}}^{\prime}=\bar{\omega}$. We choose $\mu_{\alpha^{\prime}}^{\prime \prime} \in \mathrm{B}_{N_{0}}\left(\mu ; \omega_{\alpha^{\prime}}^{\prime}\right)$. Similarly as above, $\left(\mu_{\alpha^{\prime}}^{\prime \prime}\right)_{\alpha^{\prime} \in A^{\prime}}$ as $\omega_{\alpha^{\prime}}^{\prime} \downarrow \bar{\omega}$ is contained in $\overline{\mathrm{B}}_{N_{0}}(\mu ; \omega)$, that is, $\overline{\mathrm{B}}_{N_{0}}(\mu ; \omega) \neq \phi$. The injectivity of $N_{0}$ shows that $\underline{\mathrm{B}}_{N_{0}}(\mu ; \omega)$ and $\overline{\mathrm{B}}_{N_{0}}(\mu ; \omega)$ form only one element.

Consequently, let $\mu_{\alpha}^{\prime} \in \underline{\mathrm{B}}_{N_{0}}\left(\mu ; \omega_{\alpha}\right), \quad \mu^{\prime} \in \underline{\mathrm{B}}_{N_{0}}(\mu ; \omega), \quad \mu_{\alpha^{\prime}}^{\prime \prime} \in \overline{\mathrm{B}}_{N_{0}}\left(\mu ; \omega_{\alpha^{\prime}}^{\prime}\right)$ and $\mu^{\prime \prime} \in \overline{\mathrm{B}}_{N_{0}}(\mu ; \omega)$; then $\lim _{\alpha \in \Lambda} \mu_{\alpha}^{\prime}=\mu^{\prime}$ and $\lim _{\alpha^{\prime} \in \Lambda^{\prime}} \mu_{\alpha^{\prime}}^{\prime \prime}=\mu^{\prime \prime}$.

(2) Using the complete maximum principle of $N_{0}$ and noting the above proof, we see easily (2).

(3) Let $\nu \in M_{K}^{+}(X)$ with $\nu \leqq \mu$. We choose a relatively compact open set $\omega$ in $X$ such that $\omega \supset \operatorname{supp}(\nu)$ and $N_{0} * \nu \leqq \eta$ in $\omega$. By virtue of the balayability of $N_{0}$, we can choose $\lambda \in M_{K}^{+}(X)$ such that $\operatorname{supp}(\lambda) \subset \bar{\omega}$, $N_{0} * \lambda=\eta$ in $\omega$ and $N_{0} * \lambda \leqq \eta$ on $X$. This shows that $N_{0} * \nu \leqq N_{0} * \lambda \leqq \eta$ on $X$, and $\nu$ being arbitrary, we have $N_{0} * \mu \leqq \eta$ on $X$.

(4) In the same manner as in the proof of $\underline{\mathrm{B}}_{N_{0}}(\mu ; \omega) \neq \phi$ in (1), we see that $\mathrm{E}_{N_{0}}(\omega) \neq \phi$ implies $\underline{\mathrm{E}}_{N_{0}}(\omega) \neq \phi$. For any $\gamma \in \mathrm{E}_{N_{0}}(\omega), \underline{\mathrm{E}}_{N_{0}}(\omega)=$ $\underline{\mathrm{B}}_{N_{0}}(\gamma ; \omega)$. If $\underline{\mathrm{E}}_{N_{0}}(\omega) \neq \phi$, the injectivity of $N_{0}$ shows that $\underline{\mathrm{E}}_{N_{0}}(\omega)$ forms only one element.

(5) By using the positive mass principle of $N_{0}$ and the similar method to (3), we obtain (5).

(6) Evidently $\left(\mu_{\alpha}\right)_{\alpha \in \Lambda}$ is vaguely bounded. We shall show only the latter half part. Let $\left(K_{n}\right)_{n=1}^{\infty}$ be an exhaustion of $X$. We choose $\varepsilon_{n}^{\prime} \in \mathrm{B}_{N_{0}}\left(\varepsilon, C K_{n}\right)$. The dominated convergence property of $N_{0}$ gives $\lim _{n \rightarrow \infty} N_{0} * \varepsilon_{n}^{\prime}=0$. Let 
$f \in C_{K}^{+}(X)$. Since $\left(x_{\alpha}\right)_{\alpha \in \Lambda}$ is relatively compact, $\int f d N_{0} * \varepsilon_{n}^{\prime} * \varepsilon_{x_{\alpha}} * \nu * \lambda_{2}$ converges uniformly to 0 on $\left(x_{\alpha}\right)_{\alpha \in \Lambda}$ as $n \rightarrow \infty$. Hence

$$
\varlimsup_{\alpha \in \Lambda} \int f d N_{0} * \mu_{\alpha} * \lambda_{1} \leqq \int f d N_{0} * \mu * \lambda_{1} .
$$

Using the lower semi-continuity of convolutions of non-negative Radon measures, we have $\underline{\lim }_{\alpha \in A} \int f d N_{0} * \mu_{\alpha} \geqq \int f d N_{0} * \mu$. Thus $\mu \in D^{+}\left(N_{0}\right)$ and $\lim _{\alpha \in \Lambda} N_{0} * \mu_{\alpha}=N_{0} * \mu$.

From Lemma 15 and its proof, we see the following

LEMma 16. Let $N_{0}$ be a shift-bounded Hunt convolution kernel on $X, \quad\left(\Omega_{j}\right)_{j=1}^{m}$ and $\left(\omega_{k}\right)_{k=1}^{n}$ two finite families of open sets in $X$ and let $\left(\mu_{j}\right)_{j=1}^{m} \subset D^{+}\left(N_{0}\right)$. Assume that $\mathrm{E}_{N_{0}}\left(\omega_{k}\right) \neq \phi \quad(k=1,2, \cdots, n)$. Let $\mu_{j}^{\prime} \in \underline{\mathrm{B}}_{N_{0}}\left(\mu_{j} ; \Omega_{j}\right), \gamma_{k} \in \underline{\mathrm{E}}_{N_{0}}\left(\omega_{k}\right)(j=1,2, \cdots, m ; k=1,2, \cdots, n)$ and let $\eta \in P\left(N_{0}\right)$. If $\sum_{j=1}^{m} \sum_{k=1}^{n} N_{0} *\left(\mu_{j}^{\prime}+\gamma_{k}\right) \leqq \eta$ in $\left(\cup_{j=1}^{m} \Omega_{j}\right) \cup\left(\cup_{k=1}^{n} \omega_{k}\right)$, then the same inequality holds on $X$.

Lemma 17. Let $N_{0}$ be the same as above, $\mu \in D^{+}\left(N_{0}\right)$ and let $\omega$ be an open set in $X$. For $x \in X$, we denote by $\mu_{x}^{\prime}$ and $\mu_{x}^{\prime \prime}$ the unique element in $\underline{\mathrm{B}}_{N_{0}}\left(\mu * \varepsilon_{x} ; \omega\right)$ and that in $\overline{\mathrm{B}}_{N_{0}}\left(\mu * \varepsilon_{x} ; \omega\right)$, respectively. Then we have:

(1) The mapping $x \rightarrow \mu_{x}^{\prime}$ and $x \rightarrow \mu_{x}^{\prime \prime}$ are universally measurable, that is, for any $f \in C_{K}(X)$, the functions $\int f d \mu_{x}^{\prime}$ and $\int f d \mu_{x}^{\prime \prime}$ of $x$ are universally measurable on $X$.

(2) For any $\nu \in M_{K}^{+}(X),(\mu * \nu)^{\prime} \in \underline{\mathrm{B}}_{N_{0}}(\mu * \nu ; \omega)$ and $(\mu * \nu)^{\prime \prime} \in \overline{\mathrm{B}}_{N_{0}}(\mu * \nu ; \omega)$ are of form

$$
(\mu * \nu)^{\prime}=\int \mu_{x}^{\prime} d \nu(x)^{8)} \quad \text { and } \quad(\mu * \nu)^{\prime \prime}=\int \mu_{x}^{\prime \prime} d \nu(x) .
$$

Proof. Let $x \in X$ and $\left(x_{\alpha}\right)_{\alpha \in A}$ be a net in $X$ with $x_{\alpha} \rightarrow x$. Then Lemma 15 (6) shows that $\left(\mu_{x_{\alpha}}^{\prime}\right)_{\alpha \in \Lambda}$ and $\left(\mu_{x_{\alpha}}^{\prime \prime}\right)_{\alpha \in \Lambda}$ are vaguely bounded and that every vaguely accumulation point of $\left(\mu_{x_{\alpha}}^{\prime}\right)_{\alpha \in \Lambda}$ and that of $\left(\mu_{x_{\alpha}}^{\prime \prime}\right)_{\alpha \in \Lambda}$ as $x_{\alpha} \rightarrow x$ belong to $\mathrm{B}_{N_{0}}\left(\mu * \varepsilon_{x} ; \omega\right)$. This implies that the mapping $x \rightarrow N_{0} * \mu_{x}^{\prime}$ is lower semi-continuous (i.e., for any $f \in C_{K}^{+}(X)$, the function $\int f d N_{0} * \mu_{x}^{\prime}$ is lower semi-continuous) and the mapping $x \rightarrow N_{0} * \mu_{x}^{\prime \prime}$ is upper semi-continuous. Let $\left(N_{p}\right)_{p>0}$ be the resolvent of $N_{0}$. Then, for any $p>0, x \rightarrow N_{0} * N_{p} * \mu_{x}^{\prime}$

8) This means that for any $f \in C_{K}(X), \int f d(\mu * \nu)^{\prime}=\iint f d \mu_{x}^{\prime} d \nu(x)$ 
is also lower semi-continuous and $x \rightarrow N_{0} * N_{p} * \mu_{x}^{\prime \prime}$ is also upper semicontinuous, because $N_{p}$ is also a Hunt convolution kernel on $X$, so that $N_{p}$ possesses the dominated convergence property. Hence, for any $f \in C_{K}(X)$ and any $p>0$, the resolvent equation shows that $\int f d N_{p} * \mu_{x}^{\prime}$ and $\int f d N_{p} * \mu_{x}^{\prime \prime}$ are universally measurable functions of $x$ on $X$. Since $\lim _{p \rightarrow \infty} p N_{p}=\varepsilon$ and there exists $g \in C_{K}^{+}(X)$ such that $\left|p \check{N}_{p} * f\right| \leqq \check{N}_{0} * g$ on $X$ for all $p>0$, the Lebesgue dominated convergence theorem gives $\int f d \mu_{x}^{\prime}=\lim _{p \rightarrow \infty} p \int f d N_{p} * \mu_{x}^{\prime}$ and $\int f d \mu_{x}^{\prime \prime}=\lim _{p \rightarrow \infty} p \int f d N_{p} * \mu_{x}^{\prime \prime}$, which show that $x \rightarrow \mu_{x}^{\prime}$ and $x \rightarrow \mu_{x}^{\prime \prime}$ are universally measurable.

We shall show the assertion (2). For any $f \in C_{K}^{+}(X), \iint f d \mu_{x}^{\prime} d \nu(X)$ and $\iint f d \mu_{x}^{\prime \prime} d \nu(x)$ are defined and

$$
\iint \check{N}_{0} * f d \mu_{x}^{\prime} d \nu(x) \leqq \iint \check{N}_{0} * f d \mu_{x}^{\prime \prime} d \nu(x) \leqq \int f d N_{0} *(\mu * \nu),
$$

so that $\int \mu_{x}^{\prime} d \nu(x)$ and $\int \mu_{x}^{\prime \prime} d \nu(x)$ belong to $D^{+}\left(N_{0}\right)$. We see easily that $\int \mu_{x}^{\prime} d \nu(x), \int \mu_{x}^{\prime \prime} d \nu(x) \in \mathrm{B}_{N_{0}}(\mu * \nu ; \omega)$. Let $\left(\omega_{\alpha}\right)_{\alpha \in A}$ be a net of open sets in $X$ satisfying $\bar{\omega}_{\alpha} \subset \omega_{\beta}(\alpha \supsetneqq \beta)$ and $\bigcup_{\alpha \in \Lambda} \omega_{\alpha}=\omega$. We choose $\mu_{x, \alpha}^{\prime} \in \underline{\mathrm{B}}_{N_{0}}\left(\mu * \varepsilon_{x} ; \omega_{\alpha}\right)$. Then Lemma 15 (1), (3) show that $N_{0} * \mu_{x, \alpha}^{\prime} \uparrow N_{0} * \mu_{x}^{\prime}$ as $\omega_{\alpha} \uparrow \omega$, that is,

$$
N_{0} *\left(\int \mu_{x, \alpha}^{\prime} d \nu(x)\right) \uparrow N_{0} *\left(\int \mu_{x}^{\prime} d \nu(x)\right) \quad \text { as } \quad \omega_{\alpha} \uparrow \omega
$$

This shows that $\int \mu_{x}^{\prime} d \nu(x) \in \underline{\mathrm{B}}_{N 0}(\mu * \nu ; \omega)$, and Lemma 15 (1) gives the first equality in (3.3). Let $\left(\omega_{\alpha^{\prime}}^{\prime}\right)_{\alpha^{\prime} \in \Lambda^{\prime}}$ be a net of open sets in $X$ satisfying $\omega_{\alpha^{\prime}}^{\prime} \supset \bar{\omega}_{\beta^{\prime}}^{\prime} \quad\left(\alpha^{\prime} ફ \beta^{\prime}\right)$ and $\bigcap_{\alpha^{\prime} \in \Lambda^{\prime}} \omega_{\alpha^{\prime}}^{\prime}=\bar{\omega}$. We choose $\mu_{x, \alpha^{\prime}}^{\prime \prime} \in \overline{\mathbf{B}}_{N_{0}}\left(\mu * \varepsilon_{x} ; \omega_{\alpha^{\prime}}^{\prime}\right)$. Similarly as above, we have

$$
N_{0} *\left(\int \mu_{x, \alpha^{\prime}}^{\prime \prime} d \nu(x)\right) \downarrow N_{0} *\left(\int \mu_{x}^{\prime \prime} d \nu(x)\right) \quad \text { as } \quad \omega_{\alpha^{\prime}}^{\prime} \downarrow \bar{\omega},
$$

and hence the second equality in (3.3) holds. Thus Lemma 17 is shown.

The following proposition will play an important role to prove our main theorem.

Proposition 18. Let $N_{0}$ be a shift-bounded Hunt convolution kernel on $X$ and assume that the closed subgroup generated by $\operatorname{supp}\left(N_{0}\right)$ is equal to $X$. Then, for any $0 \neq \mu \in M_{K}^{+}(X)$, there exist an open set $\omega \neq \phi$ in $X$ 
and an open neighborhood $V$ of the origin such that:

(1) For any $\mu^{\prime} \in \mathrm{B}_{N_{0}}(\mu, \omega+V)^{9)}, \int d \mu^{\prime}<\int d \mu$.

(2) $N_{0}$-equlibrium measures of $\omega$ with finite total mass do not exist.

For the poof of this proposition, we use the following result:

Lemma 19 (see [2], [4]). Let $\sigma \in M^{+}(X)$ with $\int d \sigma=1$. If a shiftbounded real Radon measure $\mu$ on $X$ satisfies $\mu=\mu * \sigma$, then, for any $x$ in the closed subgroup $\Gamma$ generated by $\operatorname{supp}(\sigma)$, we have $\mu=\mu * \varepsilon_{x}$, that is, each $x$ in $\Gamma$ is a period of $\mu$.

Proof of Proposition 18. It suffices to show the following assertion:

Let $0 \neq f \in C_{K}^{+}(X)$. Then there exist an open set $\omega \neq \phi$ in $X$ and open neighborhood $V$ of the origin such that:

$\left(1^{\prime}\right)$ For $(f \xi)^{\prime \prime} \in \overline{\mathbf{B}}_{N_{0}}(f \xi ; \omega+V), \int d(f \xi)^{\prime \prime}<\int f d \xi$.

(2') $\mathrm{E}_{N_{0}}(\omega)=\phi$, or $\mathrm{E}_{N_{0}}(\omega) \neq \phi$ and for $\gamma \in \underline{\mathrm{E}}_{N_{0}}(\omega), \int d \gamma=\infty$.

In fact, admit this assertion and let $0 \neq \mu \in M_{K}^{+}(X)$. Choose $\varphi \in C_{K}^{+}(X)$ with $\int \varphi d \xi=1$. Then there exist an open set $\omega \neq \phi$ in $X$ and an open neighborhood $V$ of the origin such that, for $f=\mu * \varphi,\left(1^{\prime}\right)$ and $\left(2^{\prime}\right)$ are verified. Since $\int d \mu=\int \mu * \varphi d \xi$, Lemma 17 (2) shows that there exists $x \in \operatorname{supp}(\varphi)$ such that for $\left(\mu * \varepsilon_{x}\right)^{\prime \prime} \in \overline{\mathrm{B}}_{N_{0}}\left(\mu * \varepsilon_{x} ; \omega+V\right), \int d\left(\mu * \varepsilon_{x}\right)^{\prime \prime}<$ $\int d \mu * \varepsilon_{x}=\int d \mu$. We remark here that $(\mu * \varphi) \xi=\int \mu * \varepsilon_{x} \varphi(x) d \xi(x)$ and for any $y \in X, \int d\left(\mu * \varepsilon_{y}\right)^{\prime \prime} \leqq \int d \mu * \varepsilon_{y}$. Put $\omega_{x}=\omega-\{x\}$ and $\mu_{x}^{\prime \prime} \in \overline{\mathrm{B}}_{N_{0}}\left(\mu ; \omega_{x}+V\right)$. Then we see easily that $\left(\mu * \varepsilon_{x}\right)^{\prime \prime}=\mu_{x}^{\prime \prime} * \varepsilon_{x}$, which implies $\int d \mu_{x}^{\prime \prime}<\int d \mu$. We remark that $\mathrm{E}_{N_{0}}(\omega)=\phi$ and $\mathrm{E}_{N_{0}}\left(\omega_{x}\right)=\phi$ are equivalent and if $\mathrm{E}_{N_{0}}(\omega) \neq \phi$, then, for $\gamma \in \underline{\mathrm{E}}_{N_{0}}(\omega)$ and $\gamma_{x} \in \underline{\mathrm{E}}_{N_{0}}\left(\omega_{x}\right), \gamma=\gamma_{x} * \varepsilon_{x}$. By the positive mass principle and Lemma $15(5)$, we see that $\omega_{x}$ and $V$ are our required open set and open neighborhood of the origin.

Dividing into the following two cases, we shall show our required assertion.

(a) Assume that there exists $0 \neq g \in C_{X}^{+}(X)$ with $\overline{\lim }_{x \rightarrow \delta} N_{0} * g(x)>0$. Then $\int d N_{0}=\infty$. Noting that $\left(N_{0} * \varepsilon_{x}\right)_{x \in X}$ is vaguely bounded, we can

9) For subsets $A, B$ of $X, A+B=\{x+y ; x \in A, y \in B\},-B=\{-x ; x \in B\}$. 
choose a net $\left(x_{\alpha}\right)_{\alpha \in \Lambda}$ in $X$ with $x_{\alpha} \rightarrow \delta$ such that $\left(N_{0} * \varepsilon_{x_{\alpha}}\right)_{\alpha \in \Lambda}$ converges vaguely and $\lim _{\alpha \in A} N_{0} * \varepsilon_{x_{\alpha}} * g(0)=\varlimsup_{\lim _{x \rightarrow \delta}} N_{0} * g(x)$. Put $\eta=\lim _{\alpha \in A} N_{0} * \varepsilon_{x_{\alpha}}$; then $\eta \neq 0$. Let $\left(N_{p}\right)_{p>0}$ be the resolvent of $N_{0}$. By the resolvent equation and $p \int d N_{p}=1(p>0)$, we have

$$
\eta=p N_{p} * \eta(p>0) .
$$

Since $\operatorname{supp}\left(N_{p}\right)=\operatorname{supp}\left(N_{0}\right)(p>0)$ and $\eta$ is shift-bounded, Lemma 19 gives $\eta=c \xi$ with some constant $c>0$. We may assume that $\int f d \xi=1$. Let $\Omega$ be a relatively compact open set with $\Omega \supset \operatorname{supp}(f)$. Since $\left(N_{0} * \varepsilon_{x} * f\right)_{x \in X}$ converges uniformly to $N_{0} * f$ on $\bar{\Omega}$ as $x \rightarrow 0$, there exists an open neighborhood $V$ of the origin such that $V=-V$, supp $(f)+\bar{V} \subset \Omega$ and for any $x \in \bar{V},\left|N_{0} * \varepsilon_{x} * f-N_{0} * f\right|<\frac{1}{3} c$ on $\bar{\Omega}$. By virtue of the complete maximum principle of $N_{0}$, we have $\left|N_{0} * \varepsilon_{x} * f-N_{0} * f\right|<\frac{1}{3} c$ on $X$ for all $x \in \bar{V}$. Put $\omega=\left\{x \in X ; N_{0} * f(x)<\frac{1}{3} c\right\}$ and $\omega=\left\{x \in X ; N_{0} * f(x)<\frac{2}{3} c\right\}$. Then $\bar{\omega}+\bar{V} \subset \omega^{\prime}$. We shall show that $\omega$ and $V$ are our required open set and open neighborhood of the origin. First we see that $\mathrm{E}_{N_{0}}(\omega)=\phi$, because, if there exists $\gamma \in \mathrm{E}_{N_{0}}(\omega)$, then $N_{0} *\left(\frac{1}{3} c \gamma+f \xi\right) \geqq \frac{1}{3} c \xi$ on $X$, which contradicts $p \int d N_{p}=1$ for all $p>0$ and $p N_{p} * N_{0} \downarrow 0$ as $p \downarrow 0$. It remains to prove that $\left(1^{\prime}\right)$ is verified. By Lemma 15 (2), it suffices to show that for any $(f \xi)^{\prime} \in B_{N_{0}}\left(f \xi ; \omega^{\prime}\right), \int d(f \xi)^{\prime}<\int f d \xi=1$. For any integer $m \geqq 1, N_{0} *(f \xi)^{\prime}$ $\leqq\left(\frac{2}{3}+1 / m\right) \eta$ in a certain open set $\supset \operatorname{supp}\left((f \xi)^{\prime}\right)$, so that Lemma 15 (3) gives $N_{0} *(f \xi)^{\prime} \leqq\left(\frac{2}{3}+1 / m\right) \eta$ on $X$. Letting $m \uparrow \infty$ and using Lemma 15 (5), we obtain $\int \mathrm{d}(f \xi)^{\prime} \leqq \frac{2}{3}$. Thus $\omega$ and $V$ are our required open set and open neighborhood of the origin.

(b) Assume that $N_{0}$ vanishes at the infinity (i.e., for any $g \in C_{K}(X)$, $\left.\lim _{x \rightarrow \delta} N_{0} * g(x)=0\right)$. Let $U_{0}$ be a relatively compact open set $\neq \phi$ in $X$ with $\bar{U}_{0} \subset\{x \in X ; f(x)>0\}$. Since $\operatorname{supp}\left(N_{0}\right) \ni 0$, we may assume that $N_{0} * f(x)>1$ on $\bar{U}_{0}$. We choose an open set $\omega_{0} \neq \phi$ and an open neighborhood $V$ of the origin such that $\bar{\omega}_{0}+\bar{V} \subset U_{0}$. Since $\lim _{x \rightarrow \delta} N_{0} * \varepsilon_{x}=0$, we can choose inductively a sequence $\left(x_{n}\right)_{n=0}^{\infty}$ in $X$ with $x_{0}=0$ and $x_{n} \rightarrow \delta$ $(n \rightarrow \infty)$ such that, for any $n \geqq 0$ and $m \geqq 0$ with $n \neq m$,

$$
N_{0} * \varepsilon_{x_{n}} * f \leqq \frac{1}{2^{|n-m|+1}} \quad \text { on }\left\{x_{m}\right\}+U_{0} \text {. }
$$

Put $U_{n}=\left\{x_{n}\right\}+U_{0}(n=1,2, \cdots)$ and $U=\bigcup_{n=1}^{\infty} U_{n}$. Evidently $\bar{U}_{m} \cap \bar{U}_{n}=\phi$ 
if $n \neq m$. Put $\omega_{n}=\left\{x_{n}\right\}+\omega_{0}(n=1,2, \cdots)$ and $\omega=\bigcup_{n=1}^{\infty} \omega_{n}$. Then $\omega+$ $V \subset U$. For any $(f \xi)^{\prime} \in \mathrm{B}_{N_{0}}(f \xi ; U)$, we set $(f \xi)_{n}^{\prime}=\left.(f \xi)^{\prime}\right|_{\bar{U}_{n}}(n \geqq 1)$. Then, by virtue of the complete maximum priciple of $N_{0}$,

$$
N_{0} *(f \xi)_{n}^{\prime} \leqq \frac{1}{2^{n+1}}\left(N_{0} * \varepsilon_{x_{n}} * f\right) \quad \text { on } X,
$$

and hence $\int d(f \xi)_{n}^{\prime} \leqq\left(1 / 2^{n+1}\right) \int f d \xi$. Consequently, $\int d(f \xi)^{\prime} \leqq \frac{1}{2} \int f d \xi$. From Lemma 15 (2), it follows that for $(f \xi)^{\prime \prime} \in \overline{\mathrm{B}}_{N_{0}}(f \xi ; \omega+V), \int d(f \xi)^{\prime \prime} \leqq \frac{1}{2} \int f d \xi$.

Let $\gamma_{n}^{\prime} \in \underline{\mathrm{E}}_{N_{0}}\left(\omega_{n}\right)$. Then $N_{0} * \boldsymbol{\gamma}_{n}^{\prime} \leqq\left(N_{0} * \varepsilon_{x_{n}} * f\right) \xi$ on $X$. For any $n \geqq 1$ and any $k$ with $1 \leqq k \leqq n$, we have, in $\omega_{k}$,

$$
N_{0} *\left(\sum_{j=1}^{n} \gamma_{j}^{\prime}\right) \leqq \xi+\sum_{j=1}^{k-1}\left(N_{0} * \varepsilon_{x_{j}} * f\right) \xi+\sum_{j=k+1}^{n}\left(N_{0} * \varepsilon_{x_{j}} * f\right) \xi \leqq 2 \xi,
$$

that is, $N_{0} *\left(\sum_{j=1}^{n} \gamma_{j}^{\prime}\right) \leqq 2 \xi$ in $\bigcup_{j=1}^{n} \omega_{j}$. This and Lemma 16 show that the same inequality holds on $X$. Thus $\sum_{n=1}^{\infty} \gamma_{n}^{\prime}$ converges vaguely. Put $\gamma^{\prime}=$ $\sum_{n=1}^{\infty} \gamma_{n}^{\prime}$; then $N_{0} * \gamma^{\prime} \geqq \xi$ in $\omega$ and $N_{0} * \gamma^{\prime} \leqq 2 \xi$ on $X$. Let $\gamma_{n} \in \underline{\mathrm{E}}_{N_{0}}\left(\bigcup_{k=1}^{n} \omega_{k}\right)$. Then $N_{0} * \gamma^{\prime} \geqq N_{0} * \gamma_{n}$ and $\sum_{k=1}^{n} N_{0} * \gamma_{k}^{\prime} \leqq 2 N_{0} * \gamma_{n}$ on $X$. By virtue of the dominated covergence property of $N_{0}$, we have $\underline{\mathrm{E}}_{N_{0}}(\omega) \neq \phi$. Let $\gamma \in \underline{\mathrm{E}}_{N_{0}}(\omega)$; then $\lim _{n \rightarrow \infty} \gamma_{n}=\gamma$. This implies that

$$
N_{0} * \gamma \leqq N_{0} * \gamma^{\prime} \leqq 2 N_{0} * \gamma \quad \text { on } X \text {. }
$$

Evidently $\int d \gamma_{n}^{\prime}=\int d \gamma_{m}^{\prime}$ for all $n \geqq 1, m \geqq 1$ and $\gamma^{\prime} \neq 0$, so that $\int d \gamma^{\prime}=\infty$. The positive mass principle of $N_{0}$ gives $\int d r=\infty$. Thus $\omega$ and $V$ are our required open set and open neighborhood of the origin.

It is a question if there exist an open set $\omega \neq \phi$ in $X$ and an open neighborhhod $V$ of the origin such that for any $0 \neq \mu \in M_{K}^{+}(X)$ with $\operatorname{supp}(\mu)$ $\subset C(\overline{\omega+V})$ and any $\mu^{\prime} \in \mathrm{B}_{N_{0}}(\mu ; \omega+V), \int d \mu^{\prime}<\int d \mu$ and $N_{0}$-equilibrium measures $\gamma$ of $\omega$ with $\int d r<\infty$ do not exist.

$\S 4$.

We return to the argument of real convolution kernels. We begin with the following

Definition 20. For a real convolution kernel $N$ on $X$ and an open set $\omega \neq \phi$ in $X$, we denote by $\operatorname{SP}_{1}(N ; \omega)$ the vague closure of 


$$
\left\{N * \mu+a \xi ; \mu \in M_{K}^{+}(X), \int d \mu=1, \operatorname{supp}(\mu) \subset \omega, a \in R\right\}
$$

and put

$$
\eta_{N, \omega}=\sup \left\{\eta \in \operatorname{SP}_{1}(N ; \omega) ; \eta \leqq N \text { on } X\right\}
$$

provided that the right hand exists in $M(X)$. If $\eta_{N, \omega}$ exists, we call it the $N$-reduced measure of $N$ on $\omega$.

Assume that $N \in(\mathrm{SMP})$. Then $\eta_{N, \omega}$ always exists and satisfies $\eta_{N, \omega}=N$ in $\omega, \eta_{N, \omega} \leqq N$ on $X$ (see Remarque 19 in [8]). Let $\left(K_{n}\right)_{n=1}^{\infty}$ be an exhaustion of $X$. Then $\left(\eta_{N, C K_{n}}\right)_{n=1}^{\infty}$ is decreasing and $\lim _{n \rightarrow \infty} \eta_{N, C K_{n}}$ is independent of the choice of $\left(K_{n}\right)_{n=1}^{\infty}$ (see $\S 3$ in [8]). Put $\eta_{N, \delta}=\lim _{n \rightarrow \infty} \eta_{N, C K_{n}}$. Then $\eta_{N, \delta}=-\infty$, i.e., for any $0 \neq f \in C_{K}^{+}(X), \lim _{n \rightarrow \infty} \int f d \eta_{N, C K_{n}}=-\infty$, or $\eta_{N, \delta} \in$ $M(X)$ (see Remarque 19 in [8]).

Proposition 9 gives immediately the following

Remark 21. Let $N \in\left(\mathrm{SMP}_{g}\right),(N, \xi) \in(\mathrm{TSMP}),\left(K_{n}\right)_{n=1}^{\infty}$ be an exhaustion of $X$ and let $\left(\varepsilon_{C K_{n}}^{\prime}, 0\right) \in \operatorname{SB}_{N}\left((\varepsilon, 0) ; C K_{n}\right)$ (see Proposition 11). Then, for any $n \geqq 2$,

$$
\eta_{N, C K_{n}} \leqq N * \varepsilon_{C K_{n}}^{\prime} \leqq \eta_{N, C K_{n-1}} \quad \text { on } X .
$$

The following proposition is shown in [8] (see Théorème 20).

Proposition 22. Let $N \in(\mathrm{SMP}),(N, \xi) \in(\mathrm{TSMP})$ and let $\left(\omega_{n}\right)_{n=1}^{\infty}$ be an open exhaustion of $X$. Then we have:

(1) For any $0<p \in R$ and any $n \geqq 1$, there exists a uniquely determined $\quad\left(\varepsilon_{p, n}^{\prime}, a_{p, n}\right) \in M_{K}^{+}(X) \times R \quad$ such that $\int d \xi_{p, n}^{\prime}=1, \operatorname{supp}\left(\varepsilon_{p, n}^{\prime}\right) \subset \bar{\omega}_{n}$, $(N+(1 / p) \varepsilon) * \varepsilon_{p, n}^{\prime}+a_{p, n} \xi=N$ in $\omega_{n},(N+(1 / p) \varepsilon) * \varepsilon_{p, n}^{\prime}+a_{p, n} \xi \leqq N$ on $X$ and for any $\nu \in M_{K}^{+}(X)$ with $\int d \nu=1$ and any $a \in R,(N+(1 / p) \varepsilon) * \nu+a \xi \geqq$ $(N+(1 / p) \varepsilon) * \varepsilon_{p, n}^{\prime}+a_{p, n} \xi$ on $X$ whenever $(N+(1 / p) \varepsilon) * \nu+a \xi \geqq N$ in $\omega_{n}$.

(2) Put $V_{p, \omega_{n}} \varepsilon=(1 / p) \varepsilon_{p, n}^{\prime}$. Then $V_{p, \omega_{n}} \varepsilon \geqq V_{p, \omega_{n+1}} \varepsilon$ in $\omega_{n}$ and $\lim _{n \rightarrow \infty} V_{p, \omega_{n}} \varepsilon$ exists.

(3) Put

$$
N_{p}=\lim _{n \rightarrow \infty} V_{p, \omega_{n}} \varepsilon\left(\in M^{+}(X)\right),
$$

then $\left(N_{p}\right)_{p>0}$ is a sub-markovian resolvent and independent of the choice of $\left(\omega_{n}\right)_{n=1}^{\infty}$. 
By using Proposition 22, we have the following

LEMmA 23. Let $N \in\left(\operatorname{SBP}_{g}\right),(N, \xi) \in(\mathrm{TSMP})$ and assume that $N$ is nonperiodic. Then there exists a uniquely determined resolvent $\left(N_{p}\right)_{p>0}$ such that

$$
N=p N * N_{p}+N_{p}
$$

Proof. First we remark that $N \in(\mathrm{SBP})$ and $N \in(\mathrm{SMP})$ are equivalent. Let $V_{p, \omega_{n}} \varepsilon, N_{p}$ and $a_{p, n}$ be the same as in Proposition 22. Then, for any $p>0$,

$$
\lim _{n \rightarrow \infty}\left((p N+\varepsilon) * V_{p, \omega_{n}} \varepsilon+a_{p, n} \xi\right)=N .
$$

Let $\left(K_{m}\right)_{m=1}^{\infty}$ be an exhaustion of $X$ with $K_{1} \ni 0$. We shall show that for any $m \geqq 2, N \neq \eta_{N, C K_{m}}$. Assume contrary that for an $m \geqq 2, N=\eta_{N, C K_{m}}$. Then Remark 21 gives $N=N * \varepsilon_{C K_{m}}^{\prime}$, where $\left(\varepsilon_{C K_{m}}^{\prime}, 0\right) \in \mathrm{SB}_{N}\left((\varepsilon, 0) ; C K_{m}\right)$. Let $\Gamma$ be the closed subgroup generated by $\operatorname{supp}\left(\xi_{C_{K_{m}}}^{\prime}\right)$; then $\Gamma \neq\{0\}$. For any $x \in X, N *\left(\varepsilon-\varepsilon_{x}\right)$ is shift-bounded (see Remarque 4 in [8]), and Lemma 19 shows that for any $y \in \Gamma, N *\left(\varepsilon-\varepsilon_{x}\right) * \varepsilon_{y}=N *\left(\varepsilon-\varepsilon_{x}\right)$. This implies that for any $x \in \Gamma$ and any integer $n \geqq 1, N-N * \varepsilon_{n x}=n\left(N-N * \varepsilon_{x}\right)$. Since for any $f \in C_{K}^{+}(X), \check{N} * f$ is upper bounded (see Remark 7 (3)), we have $\int f d\left(N-N * \varepsilon_{x}\right) \geqq 0$, and $\Gamma$ being a subgroup of $X$, we see that $N=N * \varepsilon_{x}$ for all $x \in \Gamma$. This contradicts the non-periodicity of $N$. Thus $N \neq \eta_{N, C K_{m}}$ for all $m \geqq 2$. Next we shall show that $\left(N_{p}\right)_{p>0}$ is markovian. From (4.1), $\int d \varepsilon_{C K_{m}}^{\prime}=1$ and $(p N+\varepsilon) * V_{p, \omega_{n}} \varepsilon+\mathrm{a}_{p, n} \xi \uparrow N$ as $n \uparrow \infty$, it follows that

$$
N-N * \varepsilon_{C K_{m}}^{\prime}=p\left(N-N * \varepsilon_{C K_{m}}^{\prime}\right) * N_{p}+N_{p} *\left(\varepsilon-\varepsilon_{C K_{m}}^{\prime}\right) .
$$

Assume that $\left(N_{p}\right)_{p>0}$ is not markovian. Then, for any $p>0, p \int d N_{p}<1$. From (4.4), it follows that for any $p>0$, any $n \geqq 1$ and any $m \geqq 1$,

$$
N-N * \varepsilon_{C K_{m}}^{\prime}=\left(N-N * \varepsilon_{C K_{m}}^{\prime}\right) *\left(p N_{p}\right)^{n}+\frac{1}{p} \sum_{k=1}^{n}\left(p N_{p}\right)^{k} *\left(\varepsilon-\varepsilon_{C K_{m}}^{\prime}\right),
$$

where $\left(p N_{p}\right)^{1}=p N_{p}$ and $\left(p N_{p}\right)^{n}=\left(p N_{p}\right)^{n-1} *\left(p N_{p}\right)(n \geqq 2)$. Letting $n \uparrow \infty$, we have

$$
N-N * \varepsilon_{C K_{m}}^{\prime}=\frac{1}{p} \sum_{k=1}^{\infty}\left(p N_{p}\right)^{k} *\left(\varepsilon-\varepsilon_{C K_{m}}^{\prime}\right)
$$


Since $\int d\left(\sum_{k=1}^{\infty}\left(p N_{p}\right)^{k}\right)<\infty$ and $\int d \varepsilon_{C K_{m}}^{\prime}=1$, we have $\int d\left(N-N * \varepsilon_{C K_{m}}^{\prime}\right)=0$, so that $N=N * \varepsilon_{C K_{m}}^{\prime}$. This contradicts $N \neq \eta_{N, C K_{m}}$ and $\eta_{N, C K_{m-1}} \geqq N * \varepsilon_{C K_{m}}^{\prime}$ $(m \geqq 2)$. Thus $\left(N_{p}\right)_{p>0}$ is markovian. In the same manner as in [8] (see Théorème 20 and Remarque 24), we see the rest of the proof.

Definition 24. Let $N \in(\mathrm{SMP})$. If a sub-markovian resolvent $\left(N_{p}\right)_{p>0}$ satisfying (4.2) exists, then $\left(N_{p}\right)_{p>0}$ is called the resolvent associated with $N$.

The resolvent associated with $N$ is uniquely determined if it exists (see Remarque 24 in [8]).

Lemma 25. Let $N \in(\mathrm{SMP})$ and $(N, \xi) \in(\mathrm{TSMP})$. Assume that $\eta_{N, \delta} \neq$ $-\infty, N$ is non-periodic and that the resolvent $\left(N_{p}\right)_{p>0}$ associated with $N$ exists and is markovian. Put $N^{\prime}=\eta_{N, \delta}$ and $N_{0}=N-N^{\prime}$. Then $N_{0}$ is a shift-bounded Hunt convolution kernel on $X, N_{0}=\lim _{p \rightarrow 0} N_{p}$ and every point in the closed subgroup generated by $\operatorname{supp}\left(N_{0}\right)$ is a period on $N^{\prime}$.

Proof. Let $\left(K_{n}\right)_{n=1}^{\infty}$ and $\left(\omega_{m}\right)_{m=1}^{\infty}$ be an exhaustion of $X$ and an open exhaustion of $X$, respectively. We choose $\left(\varepsilon_{n, m}^{\prime}, a_{n, m}\right) \underline{S B}_{N}\left((\varepsilon, 0) ; C K_{n} \cap \omega_{m}\right)$ whenever $C K_{n} \cap \omega_{m} \neq \phi$. Then $N * \varepsilon_{n, m}^{\prime}+a_{n, m}^{\prime} \xi \uparrow \eta_{N, C K_{n}} \quad$ as $m \uparrow \infty$ (see Remarque 19 in [8]). Here we may assume that $\left(\varepsilon_{n, m}^{\prime}\right)_{m=1}^{\infty}$ converges vaguely as $m \rightarrow \infty$. Put $\varepsilon_{n}^{\prime}=\lim _{m \rightarrow \infty} \varepsilon_{n, m}^{\prime}$; then $\int d \varepsilon_{n}^{\prime} \leqq 1$. Since $\int d N_{p}=1 / p(p>0)$, we have, for any $p>0$ and any $n \geqq 1$,

$$
\begin{aligned}
p\left(N-\eta_{N, C K_{n}}\right) * N_{p} & =\lim _{m \rightarrow \infty} p\left(N-N * \varepsilon_{n, m}^{\prime}-a_{n, m} \xi\right) * N_{p} \\
& =\lim _{m \rightarrow \infty}\left(N-N * \varepsilon_{n, m}^{\prime}-N_{p}+N_{p} * \varepsilon_{n, m}^{\prime}-a_{n, m} \xi\right) \\
& =N-\eta_{N, C K_{n}}-N_{p}+N_{p} * \varepsilon_{n}^{\prime} .
\end{aligned}
$$

Letting $n \uparrow \infty$, we have $p N_{0} * N_{p}=N_{0}-N_{p}$. Letting $p \downarrow 0$ in (4.5), we have $\lim _{p \downarrow 0} N_{p} \geqq N-\eta_{N, C K_{n}}$. Thus we see $\lim _{p \rightarrow 0} N_{p}=N_{0}$, that is, $\left(N_{p}\right)_{p>0}$ is the resolvent of $N_{0}$. Since $N$ is non-periodic, (4.2) shows that $N_{p}$ is also non-periodic $(p>0)$, which implies that $N_{0}$ is also non-periodic. Remark 14 (1), (2) show that $N_{0}$ is a shift-bounded Hunt convolution kernel. On the other hand, we have $p N^{\prime} * N_{p}=N^{\prime}$ for all $p>0$. Let $\Gamma$ be the closed subgroup generated by $\operatorname{supp}\left(N_{0}\right)$. For any $x \in X, N-N * \varepsilon_{x}$ is shift-bounded (see Remarque 4 in [8]), and $N^{\prime} \in \operatorname{SP}_{1}(N)$ gives the shiftboundedness of $N^{\prime}-N^{\prime} * \varepsilon_{x}$. Lemma 19 and $\operatorname{supp}\left(N_{0}\right)=\operatorname{supp}\left(N_{p}\right)(p>0)$ show that for any $y \in \Gamma,\left(N^{\prime}-N^{\prime} * \varepsilon_{x}\right) * \varepsilon_{y}=N^{\prime}-N^{\prime} * \varepsilon_{x}$. This implies 
that for any $x \in \Gamma$ and any integer $n \geqq 1, N^{\prime}-N^{\prime} * \varepsilon_{n x}=n\left(N^{\prime}-N^{\prime} * \varepsilon_{x}\right)$. For any $f \in C_{K}^{+}(X)$, we have $\check{N}^{\prime} * f(x) \leqq \check{N} * f(x) \leqq \sup _{y \in \operatorname{supp}(f)} \check{N} * f(y)$ on $X$. Similarly as in Lemma 23, we have $N^{\prime}=N^{\prime} * \varepsilon_{x}$ for all $x \in \Gamma$. Thus every point in $\Gamma$ is a period of $N^{\prime}$.

We shall give the proof of the "only if" part in Theorem 2. By Remark 7, it suffices to show the following

Proposition 26. If a real convolution kernel $N$ on $X$ is semi-balayable, non-periodic and satisfies $(N, \xi) \in(\mathrm{TSMP})$, then $\eta_{N, \delta}=-\infty$.

Proof. Assume contrary that $\eta_{N, \delta} \neq-\infty$. Then $\eta_{N, \delta} \in M(X)$. Put $N^{\prime}=\eta_{N, o}$ and $N_{0}=N-N^{\prime}$. We denote by $\Gamma$ the closed subgroup generated by $\operatorname{supp}\left(N_{0}\right)$. First we shall show that $N^{\prime} \in(\mathrm{SMP})$. Let $\mu, \nu \in M_{K}^{+}(X)$ with $\int d \mu=\int d \nu \neq 0$ and $a \in R$. Assume that $N^{\prime} * \mu \leqq N^{\prime} * \nu+a \xi$ in a certain open set $\omega^{\prime} \supset \operatorname{supp}(\mu)$. By Lemma 23 and Lemma 25, we have $N^{\prime} * \mu \leqq N^{\prime} * \nu+a \xi$ in $\omega^{\prime}+\Gamma$. We choose a relatively compact open set $\omega$ in $X$ such that $\omega^{\prime} \supset \bar{\omega} \supset \omega \supset \operatorname{supp}(\mu)$. Let $\left.\left(\mu^{\prime}, a^{\prime}\right) \in \mathrm{SB}_{N}(\mu, 0) ; C(\bar{\omega}+\Gamma)\right)$. Then $N * \mu^{\prime}+a^{\prime} \xi \leqq N^{\prime} * \mu$ in $C(\operatorname{supp}(\mu)+\Gamma)$. Put $c=\int d \mu$. Then $N * \mu \in \mathrm{SP}_{c}(N)$. Hence Proposition 9 gives $N * \mu^{\prime}+a^{\prime} \xi \leqq N^{\prime} * \mu$ on $X$. Evidently $N * \mu^{\prime}+a^{\prime} \xi=N^{\prime} * \mu$ in $C(\bar{\omega}+\Gamma)$. For an exhaustion $\left(K_{n}\right)_{n=1}^{\infty}$ of $X$, we choose $\varepsilon_{C K_{n}}^{\prime} \in \mathrm{B}_{N_{0}}\left(\varepsilon ; C K_{n}\right)$. Then $\operatorname{supp}\left(\varepsilon_{C K_{n}}^{\prime}\right) \subset \Gamma$ and $\int d \varepsilon_{C K_{n}}^{\prime}=1$ (see Remark 14 (2) and Lemmas 23, 25), so that

$$
N * \mu^{\prime} * \varepsilon_{C K_{n}}^{\prime}+a^{\prime} \xi \leqq N^{\prime} * \mu * \varepsilon_{C K_{n}}^{\prime}=N^{\prime} * \mu \quad \text { on } X
$$

and

$$
N * \mu^{\prime} * \varepsilon_{C K_{n}}^{\prime}+a^{\prime} \xi=N^{\prime} * \mu \quad \text { in } C(\bar{\omega}+\Gamma) .
$$

Letting $n \uparrow \infty$, we obtain that

$$
N^{\prime} * \mu^{\prime}+a^{\prime} \xi \leqq N^{\prime} * \mu \text { on } X \text { and } N^{\prime} * \mu^{\prime}+a^{\prime} \xi=N^{\prime} * \mu \text { in } C(\bar{\omega}+\Gamma) \text {, }
$$

because $\lim _{n \rightarrow \infty} N_{0} * \varepsilon_{C K_{n}}^{\prime}=0$. Hence $N^{\prime} * \mu^{\prime}=N * \mu^{\prime}$ in $C(\bar{\omega}+\Gamma)$, which shows that $\operatorname{supp}\left(N_{0} * \mu^{\prime}\right) \subset \bar{\omega}+\Gamma$. This implies $\operatorname{supp}\left(\mu^{\prime}\right) \subset \bar{\omega}+\Gamma$. On

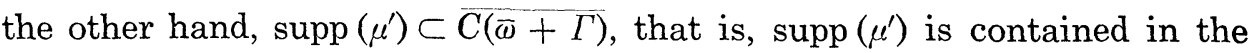
boundary $\partial(\bar{\omega}+\Gamma)$ of $\bar{\omega}+\Gamma$. Thus $N * \mu^{\prime}+a^{\prime} \xi \leqq N^{\prime} * \mu \leqq N^{\prime} * \nu+a \xi$ in $\omega^{\prime}+\Gamma \supset \operatorname{supp}\left(\mu^{\prime}\right)$, and Proposition 9 gives $N * \mu^{\prime}+a^{\prime} \xi \leqq N^{\prime} * \nu+a \xi$ on $X$. This implies $N^{\prime} * \mu \leqq N^{\prime} * \nu+a \xi$ in $C(\bar{\omega}+\Gamma)$, that is, $N^{\prime} * \mu \leqq$ $N^{\prime} * \nu+a \xi$ on $X$, which shows that $N^{\prime} \in(\mathrm{SMP})$. From $(N, \xi) \in(\mathrm{TSMP})$ and $N^{\prime} \in(\mathrm{SMP})$, we see also $\left(N^{\prime}, \xi\right) \in(\mathrm{TSMP})$. 
Evidently $N_{0}$ may be considered as a shift-bounded Hunt convolution kernel on $\Gamma$. We denote by $\xi_{\Gamma}$ a fixed Haar measure on $\Gamma$. Proposition 18 shows that, for any positive Radon measure $\mu \neq 0$ on $\Gamma$ with compact support (i.e., $\mu \in M_{K}^{+}(\Gamma)$ ), there exist an open set $\omega_{\Gamma} \neq \phi$ in $\Gamma$ and a relatively compact open neighborhood $V_{\Gamma}$ of the origin in $\Gamma$ such that:

(A) For any $\mu^{\prime \prime} \in \mathrm{B}_{N_{0}, \Gamma}\left(\mu ; \omega_{\Gamma}+V_{\Gamma}\right), \int d \mu^{\prime \prime}<\int d \mu$.

(B) $\mathrm{E}_{N_{0}, \Gamma}\left(\omega_{\Gamma}\right)=\phi$, or $\mathrm{E}_{N_{0}, \Gamma}\left(\omega_{\Gamma}\right) \neq \phi$ and for any $r \in \mathrm{E}_{N_{0}, \Gamma}\left(\omega_{\Gamma}\right), \int d \gamma=\infty$,

where $N_{0}$ being considered as a shift-bounded Hunt convolution kernel on $\Gamma, \mathrm{B}_{N_{0}, \Gamma}\left(\mu ; \omega_{\Gamma}+V_{\Gamma}\right)$ denotes the totality of $N_{0}$-balayaged measures of $\mu$ on $\omega_{\Gamma}+V_{\Gamma}$ and $\mathrm{E}_{N_{0}, \Gamma}\left(\omega_{\Gamma}\right)$ denotes the totality of $N_{0}$-equilibrium measures of $\omega_{\Gamma}{ }^{10)}$. Let $V$ be a relatively compact open neighborhood of the origin in $X$ with $\bar{V} \cap \Gamma=\bar{V}_{\Gamma}$. Put $\omega_{V}=\omega_{\Gamma}+V$; then $\omega_{V}$ is open in $X$. We choose another open neighborhood $U$ of the origin in $X$ such that $U=-U$ and $U+U \subset V$. We may consider $M_{K}^{+}(T)$ as a subset of $M_{K}^{+}(X)$. Choose $\left(\mu^{\prime}, a^{\prime}\right) \in \mathrm{SB}_{N}\left((\mu, 0) ; \omega_{V}\right)$. Then $N * \mu \geqq N * \mu^{\prime}+a^{\prime} \xi$ on $X$ implies $N^{\prime} * \mu \geqq$ $N^{\prime} * \mu^{\prime}+a^{\prime} \xi$ on $X$. Assume that $N^{\prime} * \mu-N^{\prime} * \mu^{\prime}-a^{\prime} \xi=0$. Then $N_{0} * \mu^{\prime}$ $=N_{0} * \mu$ in $\omega_{V}$ and $N_{0} * \mu^{\prime} \leqq N_{0} * \mu$ on $X$. Hence $\operatorname{supp}\left(\mu^{\prime}\right)=\bar{\omega}_{V} \cap \Gamma=$ $\left(\bar{\omega}_{\Gamma}+\bar{V}\right) \cap \Gamma=\bar{\omega}_{\Gamma}+\bar{V}_{\Gamma}$. Thus we may consider $\mu^{\prime}$ as in $M^{+}(\Gamma)$. This shows that $\mu^{\prime} \in \mathrm{B}_{N_{0}, \Gamma}\left(\mu ; \omega_{\Gamma}+V_{\Gamma}\right)$ and $\int d \mu^{\prime}=\int d \mu$, which contradicts (A). Therefore $N^{\prime} * \mu-N^{\prime} * \mu^{\prime}-a^{\prime} \xi \neq 0$. By $N^{\prime} \in$ (SMP) and Proposition 9, we have $\operatorname{supp}\left(N^{\prime} * \mu-N^{\prime} * \mu^{\prime}-a^{\prime} \xi\right) \cap \operatorname{supp}(\mu) \neq \phi$, which implies $\operatorname{supp}\left(N^{\prime} * \mu-N^{\prime} * \mu^{\prime}-a^{\prime} \xi\right) \supset \Gamma$. Let $f \in C_{K}^{+}(X)$ with $\operatorname{supp}(f) \subset U$ and $f(0)>0$. Then there eixsts $g \in C_{K}^{+}(X)$ such that $g \leqq f, g(0)>0$ and

$$
\left(N^{\prime} * \mu-N^{\prime} * \mu^{\prime}-a^{\prime} \xi\right) * f \geqq \xi_{\Gamma} * g \quad \text { on } X .
$$

Since $N_{0} * \mu^{\prime}=N_{0} * \mu+\left(N^{\prime} * \mu-N^{\prime} * \mu^{\prime}-a^{\prime} \xi\right)$ in $\omega_{V}$, we obtain that

$$
N_{0} * \mu^{\prime} * f=N_{0} * \mu * f+\left(N^{\prime} * \mu-N^{\prime} * \mu^{\prime}-a^{\prime} \xi\right) * f \quad \text { in } \omega_{\Gamma}+U .
$$

Let $\left(\omega_{\Gamma, \alpha}\right)_{\alpha \in A}$ be a net of relatively compact open sets in $\Gamma$ with $\bar{\omega}_{\Gamma, \alpha} \subset \omega_{\Gamma, \beta}$ $(\alpha \supsetneqq \beta)$ and $\bigcup_{\alpha \in \Lambda} \omega_{\Gamma, \alpha}=\omega_{\Gamma}, \gamma_{\alpha} \in \mathrm{E}_{N_{0}, \Gamma}\left(\omega_{\Gamma, \alpha}\right)(\alpha \in \Lambda)$ and let $\mu_{\omega_{\Gamma}}^{\prime \prime} \in \underline{\mathrm{B}}_{N_{0}, \Gamma}\left(\mu ; \omega_{\Gamma}\right)^{11)}$. Then, by (4.6) and (4.7), we have

10) In the case of $\mathrm{E}_{N_{0}, \Gamma}\left(\omega_{\Gamma}\right) \neq \phi$, each $\gamma \in \mathrm{E}_{N_{0}, \Gamma}\left(\omega_{\Gamma}\right)$ satisfies $\operatorname{supp}(\gamma) \subset \bar{\omega}_{\Gamma}, N_{0} * \gamma \leqq \xi_{\Gamma}$ and $N_{0} * \gamma=\xi_{\Gamma}$ on $\omega_{\Gamma}$.

11) Similarly as in the definition of $\mathrm{B}_{N_{0}}(\mu ; \omega)$, we define $\mathrm{B}_{N_{0}, \Gamma}\left(\mu ; \omega_{\Gamma}\right)$ from $\mathrm{B}_{N_{0}, \Gamma}\left(\mu ; \omega_{\Gamma}\right)$. 


$$
\begin{aligned}
N_{0} *\left(\mu_{\omega_{\Gamma}}^{\prime \prime}+\gamma_{\alpha}\right) * g & \leqq N_{0} * \mu * g+\xi_{\Gamma} * g \\
& \leqq N_{0} * \mu * f+\left(N^{\prime} * \mu-N^{\prime} * \mu^{\prime}-a^{\prime} \xi\right) * f \\
& =N_{0} * \mu^{\prime} * f \quad \text { in } \omega_{\Gamma}+U .
\end{aligned}
$$

Since $\operatorname{supp}\left(\left(\mu_{\omega_{\Gamma}}^{\prime \prime}+\gamma_{\alpha}\right) * g\right) \subset \omega_{\Gamma}+U$, the complete maximum principle of $N_{0}$ gives $N_{0} *\left(\mu_{\omega_{\Gamma}}^{\prime \prime}+\gamma_{\alpha}\right) * g \leqq N_{0} * \mu^{\prime} * f$ on $X$. Letting $\omega_{\Gamma, \alpha} \uparrow \omega_{\Gamma}$, we see, from the dominated convergence property of $N_{0}$, that there exists $\gamma \in$ $\mathrm{E}_{N_{0}, \Gamma}\left(\omega_{\Gamma}\right)$ such that

$$
N_{0} *\left(\mu_{\omega_{\Gamma}}^{\prime \prime}+\gamma\right) * g \leqq N_{0} * \mu^{\prime} * f \quad \text { on } X
$$

(see also Lemma $15(6)$ ). By the positive mass principle of $N_{0}$ (see also Lemma $15(5))$, we have $\left(\int d \mu_{\omega_{\Gamma}}^{\prime \prime}+\int d \gamma\right) \cdot \int g d \xi \leqq\left(\int d \mu^{\prime}\right) \cdot \int f d \xi$, which implies $\int d r<\infty$. This contradicts (B). The assumption $\eta_{N, \delta} \neq-\infty$ leads to this contradiction. Consequently, $\eta_{N, \delta}=-\infty$. This completes the proof.

Let $\left(\alpha_{t}\right)_{t \geqq 0}$ be a vaguely continuous convolution semi-group on $X$. It is said to be recurrent if there exists $0 \neq f \in C_{K}^{+}(X)$ with $\lim _{t \rightarrow \infty} \int_{0}^{t} \int f d \alpha_{s} d s$ $=\infty$, and it is said to be semi-transient if $\lim _{t \rightarrow \infty} \alpha_{t}=0$ and $\mu \in M_{K}^{0}(X)$, $\left(\int_{0}^{t} \alpha_{s} * \mu d s\right)_{t>0}$ is vaguely bounded.

As we mentioned in Section 1, Theorem 2 and main theorems in [8] (Théorèmes 52 and $52^{\prime}$ ) imply Theorem 1 . By Theorem 2 and a result in [8] (see Théorème 25), it can be also stated as follows:

THEOREM 27. If a real convolution kernel $N$ on $X$ is semi-balayable, non-periodic and satisfies $\inf _{x \in X} N * f(x) \leqq 0$ for all $f \in C_{K}^{0}(X)$, then there exists a uniquely determined vaguely continuous, markovian, semi-transient and recurrent convolution semi-group $\left(\alpha_{t}\right)_{t \geq 0}$ on $X$ such that for any $t>0$, $N \geqq N * \alpha_{t}$ and

$$
\lim _{t \rightarrow 0} \frac{N-N * \alpha_{t}}{t}=\varepsilon
$$

In Theorem 2 , it is a question if the condition $\inf _{x \in X} N * f(x) \leqq 0$ for all $f \in C_{K}^{0}(X)$ can be removed. By Theorem 2 and Proposition 28 in [8], we have the following

Remark 28. Assume that a real convolution kernel $N$ on $X$ satisfies the same conditions as in Theorem 27. Then, for any $\mu \in D^{+}(N)$ with 
$\int d \mu<\infty$ and any open set $\omega \neq \phi$ in $X, \underline{\operatorname{SB}}_{N}((\mu, 0) ; \omega) \neq \phi$ and it forms only one element.

In fact, it is known that if $\mu \in M_{K}^{+}(X), \underline{\mathrm{SB}}_{N}((\mu, 0) ; \omega) \neq \phi$ (see Proposition 28 in [8]). Assume that $\operatorname{supp}(\mu)$ is non-compact. Then we write $\mu=\sum_{n=1}^{\infty} \mu_{n}$, where $\mu_{n} \in M_{K}^{+}(X)$. Let $\left(\mu_{n}^{\prime}, a_{n}^{\prime}\right) \in \underline{\mathrm{SB}}_{N}\left(\left(\mu_{n}, 0\right) ; \omega\right)$. Then $a_{n}^{\prime} \leqq 0$. Let $\omega^{\prime}$ be a relatively compact open set $\neq \phi$ in $X$ with $\bar{\omega}^{\prime} \subset \omega$ and $(\nu, b) \in$ $\underline{\operatorname{SB}}_{N}\left((\mu, 0) ; \omega^{\prime}\right)$ (see Lemma 8). Then $\sum_{n=1}^{\infty} a_{n}^{\prime} \geqq b$, that is, $\sum_{n=1}^{\infty} a_{n}^{\prime}>-\infty$. This implies that $\sum_{n=1}^{\infty} \mu_{n}^{\prime} \in D^{+}(N)$. Hence we see easily that $\left(\sum_{n=1}^{\infty} \mu_{n}^{\prime}, \sum_{n=1}^{\infty} a_{n}^{\prime}\right)$. $\in \underline{\operatorname{SB}}_{N}((\mu, 0) ; \omega)$, that is, $\underline{\operatorname{SB}}_{N}((\mu, 0) ; \omega) \neq \phi$. Let $\left(\mu^{\prime}, a^{\prime}\right)$ and $\left(\mu^{\prime \prime}, a^{\prime \prime}\right)$ be in $\underline{\operatorname{SB}}_{N}((\mu, 0) ; \omega)$. Then $N * \mu^{\prime}+a^{\prime} \xi=N * \mu^{\prime \prime}+a^{\prime \prime} \xi$. Let $\left(N_{p}\right)_{p>0}$ be the resolvent associated with $N$ and $x \in X$. Since $N * \mu^{\prime} *\left(\varepsilon-\varepsilon_{x}\right)$ and $N * \mu^{\prime \prime} *\left(\varepsilon-\varepsilon_{x}\right)$ are shift-bounded, the above equality and (4.2) give

$$
N_{p} *\left(\mu^{\prime} *\left(\varepsilon-\varepsilon_{x}\right)\right)=N_{p} *\left(\mu^{\prime \prime} *\left(\varepsilon-\varepsilon_{x}\right)\right) \quad \text { for all } p>0,
$$

which implies $\mu^{\prime}-\mu^{\prime} * \varepsilon_{x}=\mu^{\prime \prime}-\mu^{\prime \prime} * \varepsilon_{x}$. Letting $x \rightarrow \delta$, we have $\mu^{\prime}=\mu^{\prime \prime}$, because $\int d \mu^{\prime}=\int d \mu^{\prime \prime}=\int d \mu<\infty$, so that $a^{\prime}=a^{\prime \prime}$. Thus $\underline{\mathrm{SB}}_{N}((\mu, 0) ; \omega)$ forms only one element.

\section{BIBLIOGRAPHY}

[ 1 ] C. Berg and G. Forst, Potential theory on locally compact abelian groups, Springerverlag, 1975.

[2] G. Choquet and J. Deny, Sur l'équation de convolution $\mu=\mu * \sigma$, C. R. Acad. Sc. Paris, 250 (1960), 799-801.

[3] - Aspects linéaires de la théorie du potentiel III. Noyaux de composition satisfaisant au principe du balayage sur tout ouvert, C. R. Acad. Sc. Paris, 250 (1960), 4260-4262.

[4] J. Deny, Sur l'équation de convolution $\mu=\mu * \sigma$, Sém. de la théorie du potentiel, 4 ème année, $n^{\circ} 5,1959 / 60$.

[5] - Noyaux de convolution de Hunt et les noyaux associés à une famille fondamentale, Ann. Inst. Fourier (Grenoble), 12 (1962), 643-667.

[6] M. Itô, Sur la famille sous-ordonnée au noyau de convolution de Hunt II, Nagoya Math. J., 53 (1974), 115-126.

[7] - Sur le principe relatif de domination pour les noyaux de convolution, Hiroshima Math. J., 5, 2 (1975), 239-350.

[8] - Une caractérisation de noyaux de convolution réels de type logarithmique, Nagoya Math. J., 97 (1985), 1-49.

Masayuki Itô

Department of Mathematics

Faculty of Sciences

Nagoya University

Chikusa-ku, Nagoya 464

Japan 
Noriaki Suzuki

Department of Mathematics

Faculty of Sciences

Hiroshima University

Naka-ku, Hiroshima 730

Japan 